\title{
A BEM formulation of free hexagons based on dynamic equilibrium
}

\author{
P. Procházka \\ Czech Technical University in Prague, Czech Republic
}

\begin{abstract}
In this paper, a time dependent (dynamical equilibrium) free hexagon DEM is formulated and solved. The main application is found in geomechanics, namely in bumps occurrence in deep mines. The time factor is included in a natural way in the model of discrete elements created by the boundary element method. One of the most important phenomena is the velocity of excavation. In the deep mines the method of depositing packs and its mechanical properties are also decisive. Their mutual coupling can principally influence the safety against bumps. For a correct understanding of the behavior of the rock aggregate (coal seam vs. overburden), the nucleation of cracks finally leading to bumps has to be treated as time dependent, while so far it was observed only from statical equilibrium. According to new experiments and results from accessible literature, a dynamical effect has to be included in thr formulation.

Contact problems leading to bumps occurrence in deep mines have been solved in many of the papers of the present author for the static case. Either Lagrangian multipliers or penalty formulation were used. The new formulation has to be submitted in terms of a penalty, which if high enough (bond effect of adjacent elements) suppresses the influence of time. By including the interface properties with the lumped inertia mass of the elements, complex nucleation can be studied and the information on possible rock bursts is improved. From some examples it was shown in the static case that the behavior at the face of longwall mining is close to that near the crack tip, and the differences in material properties of coal and overburden are also not negligible. These factors are also expected to be important in the case of dynamic problems. Some examples show the application of the procedure proposed.

Keywords: discrete element method, boundary element method, dynamical equilibrium.
\end{abstract}




\section{Introduction}

In this paper we discuss a possible solution of the stability of side walls for longwall mining in deep mines. Two phase medium is modeled: rock mass compresses a coal seam, which is positioned at a depth exceeding $700 \mathrm{~m}$.

Numerical methods seem to be the cheapest tool for assessing different types of structures. In the case of the description of underground massif often PFC (particle flow code) [1, 2], is used, which has a long lasting tradition, but for prediction of rock bumps it is a very poor tool. This is caused by the fact that the PFC starts with dynamical equilibrium so that the process described by the PFC is dynamic, which is in contradiction with the real behavior of the rock before bumps occurrence. Moreover, only a one-point touch of adjacent elements cannot describe the true distribution on element boundary displacements and tractions needed for description of continuum. From these considerations it follows that prediction by the PFC has a very poor chance in planning longwall mining.

If the theory of damage should be involved into formulation of the problem to be solved, special treatment is required using continuous methods (FEM, BEM, etc.). The methods, which are extensively used, start with realization of the trial body as a continuum. Named here "Cohesive zone method" [3], "Manifold method" [4-6], for example, which deal with Barenblatt's theory. In the problem of rock bursts such methods are on one side uneasy applicable and on the other side exhibit unreal behavior, according to a couple of test examples. "Smooth Hydrodynamics Method" [7] seems more promising, but problems occur with introducing general boundary conditions

This is why test experiments have been carried out to get knowledge about a reasonable approach for solving the problem. One of a possible experimental treatment was suggested in paper [8], where Araldit and physically similar materials are used. Based on models of such a similarity conception coupled modeling was used in [9-11]. In [9] rock bursts are studied in mines at extreme depths. The free hexagon method is used to determine the bumps state in the rock. In [10] a similar problem is solved for more general cases occurring during mining. In [11] tunnel face stability is assessed. The scale model used in this paper is prepared in collaboration with Muenchen University; Lippman was the coordinator together with J. Vacek.

In [12-14] results from on site measurements are published, recommendations are provided on how to proceed in determining the rock bursts and, what is the most important to us, the way of movement is shown in these publications.

The free hexagon method seems to be one being very promising, as the result from experiments and numerical models are reasonably comparable. The static equilibrium was used in formulation of the free hexagon method. This method has been established in the middle of 90-ties and the fundaments can be found in $[15,16]$. In [17] the method is applied to the stability of a tunnel face. The nucleation of cracking is observed at the face, but no larger displacements were calculated; only the failure state was determined. A concise formulation of two 
methods, static PFC and the free hexagon method, is published in [16] together with couple of applications to various geotechnical problems.

In this paper interface between statical and dynamical states before and at the moment of bumps is characterized. First, the method will be described and basic formulas will be derived, and then some applications to rock bumps will be presented. Time-dependent problem with the D'Alembert forces, which are caused by contact forces of moving particles, simplifies the body of the earth (soil) to a set of hexagons, which are, or are not in mutual contact. The material properties of the hexagons are determined from the state of stresses. The hexagons represent a possible shape of grains the earth consists of. The model proposed in this paper may, contrary to modern numerical methods (FEM, BEM, etc.), enables one to disconnect the medium described by the hexagons, when needed (e.g. providing certain requirement on tensile strength is violated). The most natural contact conditions (Mohr-Coulomb hypotheses) may be simply introduced and, after imposing all such of those contact conditions, the localized damage, or "cracking" can be found out. The stability then depends on the "measure" of the touched zone.

Mechanical behavior inside each element is either linear or non-linear (plastic, viscoelastic, viscoplastic, etc). To describe such behavior, boundary elements are applied. In some papers FEM is used, which causes far more difficulties then the BEM, see, e.g., [17], where tractions are by one degree of polynomials lesser then displacements, although along the boundary abscissas linear relation traction $\mathrm{x}$ displacement holds. This is in contradiction with the assertion that along the adjacent boundaries of the elements the tractions and the displacements have the same degree of approximations (splines). On the other hand, it is typically fulfilled when using the BEM that the element boundary displacements and tractions are of the same degree of approximation.

A typical coupled modeling (mathematical and experimental) is published in [18]. In the latter publication the experimental models are based on scale modeling and created from physically equivalent materials. In our case similar modeling is used with such an exception that very particular materials are used, see [7-11].

\section{Basic assumptions}

Starting with statical equilibrium in the first stage of excavation, after dislocations in the rock continuum and in the coal seam that appears, time dependent dynamical equilibrium has to be considered. Under the assumption that the material properties of both rock and coal are known, hexagon elements are created and linear behavior in them is supposed. Since the elements are considered to be small enough, isotropic case is taken into account, i.e. the elements are homogeneous and isotropic with material characterization given by modulus of elasticity $E$ and Poisson's ratio $v$, for example. Classical problem involving generalized Coulomb's friction and exclusion of tensile stress exceeding the tensile strength along the interfaces (possible dislocations) is solved. Typical set up of adjacent elements is illustrated in Fig. 1. In what 
follows the distribution of mass inside each element is neglected in such a sense that it is concentrated at c.o.g. of the element. Then, first the solution of elastic problem in an element is formulated and the element is put into the neighborhood of adjacent elements. Regular distribution of elements is assumed, i.e. only one matrix relating tractions and boundary displacements will be provided.

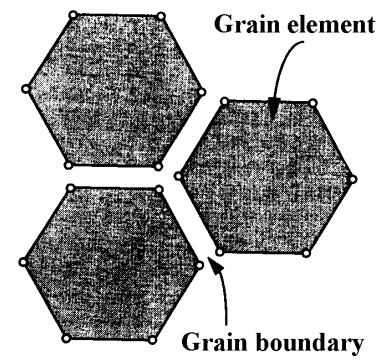

Figure 1: $\quad$ Adjacent grains set up.

\section{Boundary element solution in one hexagon}

The solution of elasticity on each hexagonal element $\Omega$ is approximated by concentration of DOFs to vertices of the hexagon, and distribution of boundary displacements and tractions along edges $\Gamma_{s}, s=1, \ldots, 6$ of the hexagon $\Omega$ is assumed to be linear. Then, generally, integral equations formulate the problem:

$$
c_{i k} u_{k}(\boldsymbol{\xi})=\int_{\Gamma_{s}} p_{i}(\boldsymbol{x}) u_{i k}^{*}(\boldsymbol{x}, \boldsymbol{\xi}) \mathrm{d} \boldsymbol{x}-\int_{\Gamma_{s}} u_{i}(\boldsymbol{x}) p_{i k}^{*}(\boldsymbol{x}, \boldsymbol{\xi}) \mathrm{d} \boldsymbol{x}+\int_{\Omega} b_{i}(\boldsymbol{x}) u_{i k}^{*}(\boldsymbol{x}, \boldsymbol{\xi}) \mathrm{d} \boldsymbol{x}
$$

where $i$ and $k$ run 1,2 , and $s=1, \ldots, 6$. In case of regular element distribution is considered, $\delta_{i k}$ is Kronecker's delta. In case the regular hexagons are used and linear distribution of both displacements and tractions is used, $c_{i k}=\frac{1}{3} \delta_{i k}$, and then

$$
\frac{1}{3} \delta_{i k} u_{k}(\boldsymbol{\xi})=\int_{\Gamma_{s}} p_{i}(\boldsymbol{x}) u_{i k}^{*}(\boldsymbol{x}, \boldsymbol{\xi}) \mathrm{d} \boldsymbol{x}-\int_{\Gamma_{s}} u_{i}(\boldsymbol{x}) p_{i k}^{*}(\boldsymbol{x}, \boldsymbol{\xi}) \mathrm{d} \boldsymbol{x}+\int_{\Omega} b_{i}(\boldsymbol{x}) u_{i k}^{*}(\boldsymbol{x}, \boldsymbol{\xi}) \mathrm{d} \boldsymbol{x}
$$

while for uniform distribution of both geometrical and statical characterizations along the boundaries we get

$$
\frac{1}{2} \delta_{i k} u_{k}(\boldsymbol{\xi})=p_{i} \int_{\Gamma_{s}} u_{i k}^{*}(\boldsymbol{x}, \boldsymbol{\xi}) \mathrm{d} \boldsymbol{x}-u_{i} \int_{\Gamma_{s}} p_{i k}^{*}(\boldsymbol{x}, \boldsymbol{\xi}) \mathrm{d} \boldsymbol{x}+\int_{\Omega} b_{i}(\boldsymbol{x}) u_{i k}^{*}(\boldsymbol{x}, \boldsymbol{\xi}) \mathrm{d} \boldsymbol{x}
$$




$$
k=1,2, s=1, \ldots, 6,
$$

Formula (1b) is used in this text, i.e. uniform distribution is applied. Knowing the form of kernels denoted by asterisk and substituting approximations for boundary displacements and tractions, matrix equations are obtained:

$$
A \boldsymbol{u}=\boldsymbol{B p}+\boldsymbol{b}, \quad \boldsymbol{K u}=\boldsymbol{p}+\boldsymbol{V}
$$

where $\boldsymbol{A}, \boldsymbol{B}$ and $\boldsymbol{K}$ are square matrices $(12 * 12), \boldsymbol{u}$ is the vector of displacement approximations at vertices, $\boldsymbol{p}$ that of tractions and $\boldsymbol{b}$ and $\boldsymbol{V}$ are vectors of volume weight influences. The latter are vectors $(1 * 12)$.

\section{Statical contact conditions}

Let us consider two hexagons being in possible contact, see Fig. 2. Introduce a pseudo-cone $K$, which is defined as:

$$
\begin{aligned}
& K \equiv\left\{\boldsymbol{u} \in V,[u]_{n} \geq 0, p_{n} \leq p_{n}^{+}, \text {if } p_{n} \geq p_{n}^{+} \Rightarrow p_{n}=0,\right. \\
& \left|p_{t}\right| \leq c \kappa\left(p_{n}^{+}-p_{n}\right)-p_{n} \tan \varphi, \\
& \text { if } \left.\left|p_{t}\right| \geq c \kappa\left(p_{n}^{+}-p_{n}\right)-p_{n} \tan \varphi \Rightarrow p_{t}=p_{n} \tan \varphi \operatorname{sgn}[u]_{t}\right\}
\end{aligned}
$$

where $[u]_{n}=u_{n}^{2}-u_{n}^{1},[u]_{t}=u_{t}^{2}-u_{t}^{1}, \boldsymbol{u}$ is split into normal $u_{n}$ and tangential (shear) $u_{t}$ components, $\boldsymbol{n}$ is unit outward normal with respect to element $1, V$ is admissible space of displacements, traction $\boldsymbol{p}$ has now components $\left\{p_{n}, p_{t}\right\}$, i.e. projections to normal and tangential directions, $p_{n}^{+}$is the tensile strength, $c$ is the cohesion or shear strength, and $\varphi$ is the angle of internal friction of the material (rock, coal), $\kappa$ is the Heaviside function being equal to one for positive arguments and zero otherwise. Here strict sign convention is used: positive sign is tension, while negative one means compression. The pseudo-cone $K$ becomes a cone for $p_{n}^{+}=0$ and frictionless case.

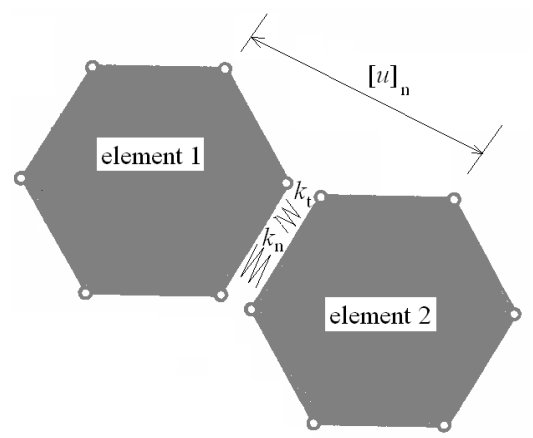

Figure 2: Two hexagons in possible contact. 


\section{Fischera's conditions}

Fischera's conditions have been formerly formulated for $K$ being a cone. In our case the conditions in normal direction can be written as:

$$
p_{n}^{+} \kappa\left(p_{n}^{+}-p_{n}\right)-p_{n} \geq 0, \quad[u]_{n} \geq 0,\left\{p_{n}^{+} \kappa\left(p_{n}^{+}-p_{n}\right)-p_{n}\right\}[u]_{n}=0,
$$

Similarly, in the tangential direction it holds:

$$
\begin{gathered}
c \kappa\left(p_{n}^{+}-p_{n}\right)-p_{n} \tan \varphi-\left|p_{t}\right| \geq 0, \quad\left|[u]_{t}\right| \geq 0, \\
\left\{c \kappa\left(p_{n}^{+}-p_{n}\right)-p_{n} \tan \varphi-\left|p_{t}\right|\right\}\left|[u]_{t}\right|=0,
\end{gathered}
$$

The energy of the system can be stored as:

$$
\begin{aligned}
\Pi=\frac{1}{2} \sum_{\alpha=1}^{N} a_{\alpha}(\boldsymbol{u}, \boldsymbol{u})-\int_{\Gamma}^{-\mathrm{T}} \boldsymbol{u} \mathrm{d} \boldsymbol{x}-\sum_{\beta=1}^{n} \int_{\Gamma_{\beta}}\left\{\left(p_{n}^{+}\right)^{\beta} \kappa\left(p_{n}^{+}-p_{n}^{\beta}\right)-p_{n}^{\beta}\right\}[u]_{n}^{\beta} \mathrm{d} \boldsymbol{x}- \\
\quad-\sum_{\beta=!}^{n} \int_{\Gamma_{\beta}}\left\{c^{\beta} \kappa\left(p_{n}^{+}-p_{n}^{\beta}\right)-p_{n}^{\beta} \tan \varphi-\left|p_{t}^{\beta}\right|\right\}\left|[u]_{t}^{\beta}\right| \mathrm{d} \boldsymbol{x}
\end{aligned}
$$

where $\alpha$ runs over all hexagon elements, $\alpha=1, \ldots, N, \beta$ runs all contact edges of possible contacts $\Gamma_{\beta}, \beta=1, \ldots, n, \Gamma$ is the external boundary where $\boldsymbol{p}$ is prescribed, and

$$
a_{\alpha}(\boldsymbol{u}, \boldsymbol{u})=\int_{\Omega_{\alpha}}\left(\boldsymbol{\sigma}^{\alpha}\right)^{\mathrm{T}} \boldsymbol{\varepsilon}^{\alpha} \mathrm{d} \boldsymbol{x}
$$

is the internal energy (bilinear form) inside a hexagon $\Omega_{\alpha}, \boldsymbol{\sigma}^{\alpha}, \boldsymbol{\varepsilon}^{\alpha}$ are respectively stresses and strains in $\Omega_{\alpha}$.

\section{Penalty formulation}

Setting $p_{n}=k_{n}[u]_{n}, \quad p_{t}=k_{t}[u]_{t}$, where $k_{n}, k_{t}$ are normal spring and tangential spring stiffnesses, and substituting these expressions in (6) yields

$$
\begin{gathered}
\Pi=\frac{1}{2} \sum_{\alpha=1}^{N} a_{\alpha}(\boldsymbol{u}, \boldsymbol{u})-\int_{\Gamma}^{-\mathrm{p}} \boldsymbol{u} \mathrm{d} \boldsymbol{x}+ \\
+\sum_{\beta=1}^{n} \int_{\Gamma_{\beta}}\left\{k_{n}^{\beta}\left([u]_{\mathrm{n}}^{\beta}\right)^{2}+k_{n}^{\beta}[u]_{\mathrm{n}}^{\beta}\left|[u]_{t}^{\beta}\right|+k_{t}^{\beta}\left([u]_{t}^{\beta}\right)^{2}\right\} \mathrm{d} \boldsymbol{x}- \\
-\sum_{\beta=!}^{n} \int_{\Gamma_{\beta}}\left\{\left(p_{n}^{+}\right)^{\beta} \kappa\left(p_{n}^{+}-p_{n}^{\beta}\right)[u]_{n}^{\beta}+c^{\beta} \kappa\left(p_{n}^{+}-p_{n}^{\beta}\right)\left|[u]_{\mathrm{t}}^{\beta}\right|\right\} \mathrm{d} \boldsymbol{x}
\end{gathered}
$$




\section{Dynamical response}

If each hexagonal element is considered small enough, lump mass dynamical problem can be formulated according to Fig. 2, where for the sake of simplicity the influence of rotation is neglected. Suppose the element 1 possesses a stable position, then element 2 will obey differential equation

$$
m \frac{\mathrm{d}^{2}}{\mathrm{~d} t^{2}} w+k_{n} w=0, \quad w=[u]_{n}
$$

where $m$ is a mass of the element 1 , measured in $\mathrm{kg}$. From the latter equation immediately follows that if $k_{n}$ is large the inertia forces are suppressed and in each small enough time step no dynamical influence occurs. This assertion will be précised in the next text. The solution of latter equation is known as:

$$
w(t)=w_{0} \frac{\sin \omega \bar{\xi}}{\sin \omega}+w_{1} \frac{\sin \omega \xi}{\sin \omega}, \quad \xi=\frac{t-t_{0}}{h}, \quad \omega=h \sqrt{\frac{k_{n}}{m}}, \quad \bar{\xi}=1-\xi
$$

where $h=t_{1}-t_{0}$ is the time step, $w_{0}=w\left(t_{0}\right), w_{1}=w\left(t_{1}\right), t_{0}$ is the initial time, $t_{1}$ is the time in the next time step. At the middle of the time interval, the value of displacement $w$ and the first derivative by time $t$ are derived as:

$$
w_{\frac{1}{2}}=w\left(\xi=\frac{1}{2}\right)=\frac{w_{0}+w_{1}}{2 \cos \frac{\omega}{2}}, \frac{\mathrm{d}}{\mathrm{d} t} w_{\frac{1}{2}}=\frac{\omega\left(w_{1}-w_{0}\right)}{2 h \sin \frac{\omega}{2}}
$$

From equations (10) and (11) it follows an important bound estimate on the time step $h: h \leq \frac{\pi}{2} \sqrt{\frac{m}{k_{n}}}$. The only troublesome point remains for $k_{n} \rightarrow 0$. Then linear relation follows from the governing equation and, consequently, the velocity is constant. This is in compliance with the D'Alembert law. The last inequality leads us also to the fact that in case of large penalty $k_{n}$ no differences in displacements can be expected due to inertia forces.

Using well known approximation formula for second derivative and the above approximate formulas we get:

$$
\frac{\mathrm{d}^{2}}{\mathrm{~d} t^{2}} w\left(\xi=\frac{1}{2}\right)=\frac{1}{4 h^{2}}\left[w(\xi=1)-2 w\left(\xi=\frac{1}{2}\right)+w(\xi=0)\right]
$$

which is an explicit formula for calculating $w(\xi=1)$. Using vector projection to the coordinates system, resulting movement is received. At the moment the c.o.g. of the element is then moved assuming the deformed body as rigid. 


\section{Examples}

A study of a longwall mine has been carried out. Material coefficients of the rock massif $\Omega$ have the following values: $E=52500 \mathrm{MPa}, v=0.29$, the peak values $E_{\mathrm{p}}=38000 \mathrm{MPa}$, and $v_{\mathrm{p}}=0.38$, the residual values $E_{\mathrm{r}}=5000 \mathrm{MPa}$, the angle of internal friction is 42 degrees, its residual value is 32 degrees, the shear strength $c=0.9 \mathrm{MPa}$ and its residual value is considered as $0.4 \mathrm{MPa}$. The coal seam is brittle, with $E=5500 \mathrm{MPa}, v=0.39$, the angle of internal friction and the shear strength vary. In Fig. 3 setting of hexagonal elements is seen, Figs. 4 and 5 display the movements at $t=0.1 \mathrm{sec}, h=0.001 \mathrm{sec}$, i.e. the starting spring stiffness $k_{n}$ is derived as $10^{10}$.

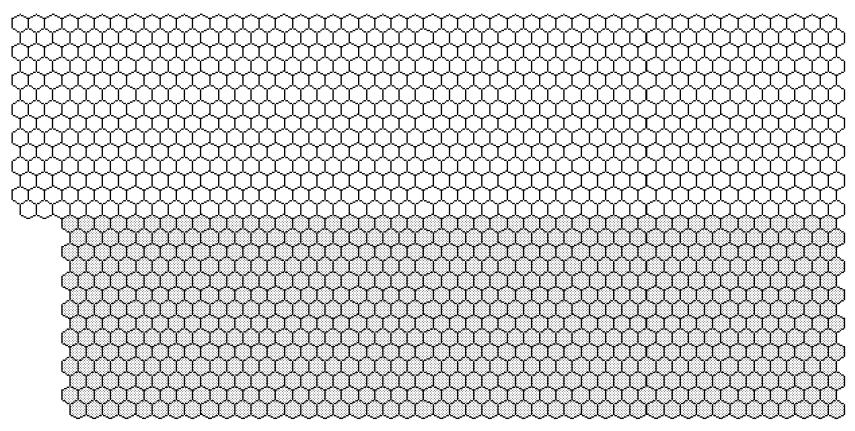

Figure 3: $\quad$ Setting of the hexagonal elements.

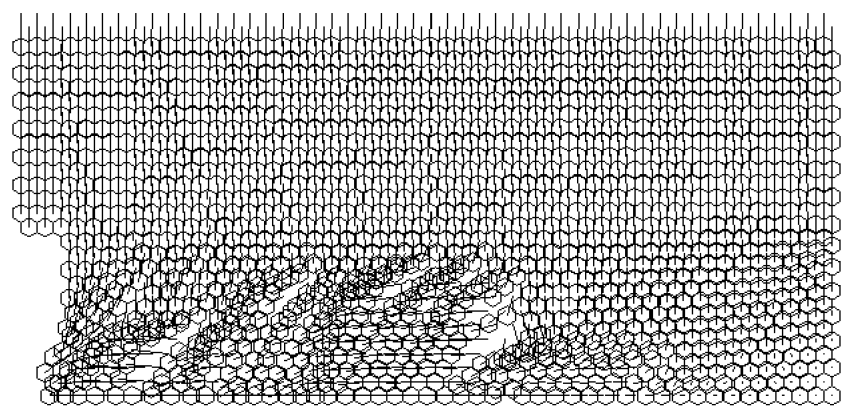

Figure 4: $\quad$ Movements for $c=100 \mathrm{kPa}$ and $p_{n}^{+}=10 \mathrm{kPa}$. 


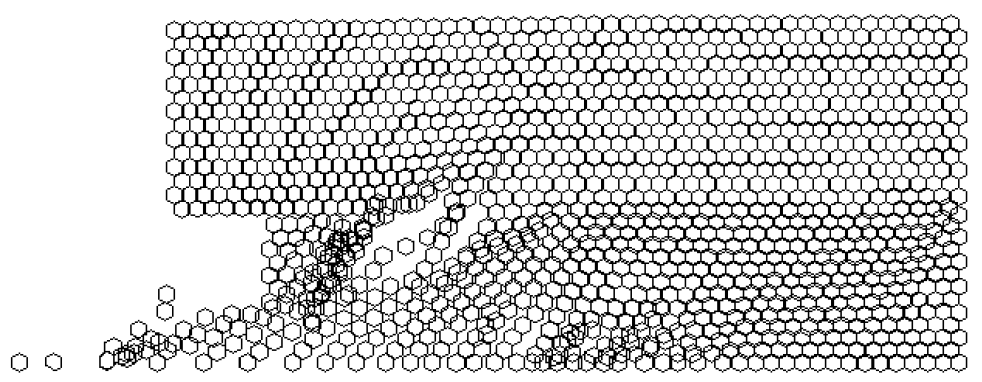

Figure 5: $\quad$ Movements of the particles for $c=10 \mathrm{kPa}$ and $p_{n}^{+}=25 \mathrm{kPa}$

\section{Conclusions}

Dynamical behavior of the face of a coal seam at the moment of rock burst and closely after it is studied in this paper. In comparison with the PFC we start with a different shape of particles (to have the possibility to also get stresses in the particles) and with static equilibrium in the state when no bumps occur. After nucleation of cracks, or in other words if small movements are observed, the kinetics of the moved particles is considered. The inertia part of the governing equation starts to prevail and be active. Generally, in contradiction with the PFC dynamical equilibrium is taken into consideration after enough movement of the hexagonal elements. The forces induced along the boundaries of adjacent particles or after mutual touching of extruded particles cause an acceleration of the particles, which defines the way of movement due to D'Alembert forces. Influence of spring stiffness stabilizes the iterative process. If removed, the process can degenerate in unstable convergence of diverge at all.

\section{Acknowledgement}

Financial support by GACR, project No. 103/05/0334 is appreciated.

\section{References}

[1] Cundall, P.A. A computer model for simulation progressive large scale movements of blocky rock systems. Symposium of the international society of rock mechanics, 132-150, 1971.

[2] Moreau, J.J. Some numerical methods in multibody dynamics: Application to granular materials. Eur. J. Mech. Solids, 13, 4, 1994, 93114.

[3] Elices, M., Guinea, G.V., Gomez, J. \& Planas, J. The cohesive zone model: advantages, limitations and challenges. Engineering Fracture Mechanics 69, 2002, 137-163. 
[4] Babuska, I., Melenk, J.M.: The partition of unity method, Int. J. Numer. Meth. Engrg. 40 (1997) 727-758

[5] Chen, G., Ohnoshi, Y., Ito, T. Development of high-order manifold method, Int. J. Numer. Meth. Engrg. 43 (1998) 685-712

[6] Lin, J.S.: A mesh-based partition of unity method for discontinuity modeling, Comput. Meth. Appl. Mech. Engrg. 192 (2003) 1515-1532

[7] Zhu, W.C., Tang, C.A.: Micromechanical Model for Simulating the Fracture Process of Rock, Rock Mech. And Rock Engrg 37 (1), 25-56.

[8] Kuch, R., Lippmann, H. \& Zhang, J. Simulating coal mine bumps with model material. Rockbursts and seismicity in mines, Gibowitz \& Lasocki (eds.), Balkema, Rotterdam, 1997, 23-25.

[9] Vacek, J. \& Procházka, P. Behaviour of Brittle Rock in Extreme Depth. Our World in Concrete \& Structures. Singapore: CI-Premier, 19, 2000, 653-660.

[10] Vacek, J. \& Procházka, P. Rock Bumps Occurrence during Mining. Computational Methods and Experimental Measurements X. Southampton: WIT Press, 2001, 437-446.

[11] Procházka, P. \& Vacek, J. Comparative Study of Tunnel Face Stability. Damage \& Fracture Mechanics VII. Southampton: WIT Press, 2002, 163172.

[12] Haramy, K.Y. and Morgan, T.A. \& DeWaele, R.E. A method for estimating western coal strengths from point load tests on irregular bumps. 2nd Conf. on Ground Control in Mining, West Virginia University, July 19-21, 1982, 123-136.

[13] Haramy, K.Y., Magers, J.A. \& McDonnell, J.P. Mining under strong roof. 7 th Int. Conf. on Ground Control in Mining, Bureau of Mines, Denver, USA, 1992, 179-194.

[14] Harami, K.Y. \& Brady, B.T. A methodology to determine in situ rock mass failure. Internal report of Bureau of Mines, Denver, CO, USA, 1995.

[15] Procházka, P. \& Válek, M. The BEM Formulation of Distinct Element Method. BETECH XXII. Cambridge: WIT Press, 2000, 395-404.

[16] Procházka, P. Application of discrete element methods to fracture mechanics of rock bursts. Engng. Fract. Mech. 2003.

[17] Onck, P. \& van der Giessen, E. Growth of an initially sharp crack by grain boundary cavitation. JMPS 1999, 523-542.

[18] Procházka, P. \& Trčková, J. Coupled modeling of Concrete Tunnel Lining. Our World in Concrete and Structures, Singapore, 2000, 125-132.

[19] Procházka, P. \& Válek, M. Stability of Tunnel Face Using Coupled DSC \& TFA Models. Damage and Fracture Mechanics VI. Montreal, WIT Press, 2000, 471-480.

[20] Brebbia, C.A., Teles, J.C.F. and Wrobel, L.C. Boundary element techniques. Springer Verlag, Berlin, Heidelberg, NYC, 1984. 